\title{
Enhancement of Carbon Fiber Cloth Reactivity to Air by Potassium Addition using Ion Exchange
}

\author{
P. L. Walker, Jr., T. A. Eapen, I. M. Ismail, and O. P. Mahajan* \\ Department of Materials Science and Engineering \\ The Pennsylvania State University \\ University Park, PA, 16802
}

(Received March 23, 1981)

\begin{abstract}
Enhancement of the reactivity to air of polyacrylonitrile-based carbon fiber cloth has been achieved by the addition of metals to the cloth. Prior to metal addition, the cloth was oxidized in $54 \mathrm{wt} \%$ nitric acid in order, both, to increase the surface area of the cloth and to add carboxyl groups to the surface. Metal addition was then achieved by soaking the cloth in metal acetate solution to effect exchange between the metal cation and hydrogen on the carboxyl groups. The addition of potassium, sodium, calcium, and barium enhanced fiber cloth reactivity to air at $573 \mathrm{~K}$. Extended studies using potassium addition showed that success in enhancing fiber cloth reactivity to air depends on: extent of cloth oxidation in nitric acid, time of exchange in potassium acetate solution, and the thoroughness of removing metal acetate from the fiber pore structure following exchange. With increased potassium loading on fiber cloth which has undergone a fixed extent of prior oxidation, cloth reactivity in air reaches a maximum and in some cases decreases. Optimum loading is associated with potassium added by exchange; additional loading is associated with potassium added by decomposition of acetate not removed from the fiber pore system by washing. Cloth reactivity increases essentially linearly with increase in potassium addition via exchange.
\end{abstract}

\section{INTRODUCTION}

Concern has arisen over the potential for accidental release of carbon fibers from resin matrix composites should the composite become involved in a fire ${ }^{1)}$. Once released from the resin matrix which is consumed in the fire, the light and fine carbon fibers can be carried in the atmosphere over large distances. Because of their high electrical conductivity, falling out of carbon fibers onto unprotected electrical and electronic circuits can cause a variety of problems, including resistive loading, temporary short circuits, and arcing. Thus for some applications there is interest in enhancing the reactivity of carbon fibers to air so that they will be consumed, along with the resin matrix, in a fire.

It is known that reactivity of carbon to oxidizing gases can be increased by increasing the active

* Present Address: Corporate Research, Standard Oil of Indiana Research Center, Naperville, IL, 60540 . surface area (ASA) of the carbon and by adding inorganic catalysts to the carbon ${ }^{2,3)}$. There have been a number of studies on increasing the surface area of carbon fibers using oxidative etching in order to subsequently enhance their bonding to the resin matrix ${ }^{4}$. Probably the use of nitric acid has been the most common ${ }^{4 \sim 7)}$. Until recently, inorganics have been added to carbon primarily by impregnation of the carbon with a salt solution, followed by drying, and decomposition of the salt $^{3)}$. More recently, it has been shown that well dispersed inorganics (cations) can be effectively added to carbons using ion exchange with the hydrogen on carboxyl groups ${ }^{8}$. This has been the approach used in this study in an attempt to enhance fiber reactivity to air.

\section{EXPERIMENTAL STUDIES}

\subsection{Materials}

Studies were made on carbon fiber cloth woven by Woven Structures Inc. from T-300 carbon fiber yarn grade WYP 30 1/0 manufactured by Union 
Table 1 Selected properties of fibers in T-300 carbon cloth.

\begin{tabular}{lc}
\hline Surface Area, $\mathrm{m}^{2} / \mathrm{g}$ & 0.37 \\
$L_{c}, \mathrm{~nm}$ & 1.1 \\
Interlayer spacing, nm & 0.356 \\
Hg density, g/cc & 1.71 \\
X-ray density, g/cc & 2.14 \\
He density, g/cc & 1.79 \\
Total Porosity, cc/g & 0.117 \\
Open Porosity, cc/g & 0.026 \\
Closed Porosity, cc/g & 0.091 \\
\hline
\end{tabular}

Carbide $^{9)}$. The polyacrylonitrile-based (PAN) yarn consisted of 3000 filaments ( $7 \mu \mathrm{m}$ in diameter) in a one-ply construction. The surface was treated with about $1.0-1.5 \mathrm{wt} \%$ proprietary sizing. Selected properties of the fibers in the cloth are given in Table 1 . The small crystallite size $\left(L_{c}\right)$ of the fiber cloth and its high nitrogen content (that is, $6.5 \mathrm{wt} \%)$ is consistent with its having been heated to less than $1800 \mathrm{~K}^{10,11)}$. Preferential basal plane alignment along the fiber axis results in a low surface area for the fibers, as measured from $\mathrm{Kr}$ adsorption at $77 \mathrm{~K}$ (using the BET equation) and a small amount of porosity accessible to helium at room temperature. In fact, the $\mathrm{Kr}$ surface area of $0.37 \mathrm{~m}^{2} / \mathrm{g}$ agrees well with the geometric area of the filaments $\left(0.33 \mathrm{~m}^{2} / \mathrm{g}\right)$ based on a diameter of $7 \mu \mathrm{m}$ and density of $1.71 \mathrm{~g} / \mathrm{cc}$. It is significant that the fibers had a substantial total pore volume as calculated from $\mathrm{x}$-ray and mercury densities, that is a total porosity of $20 \%$, but that about $78 \%$ of the total pore volume was inaccessible to helium.

A semi-quantitative spectroscopic analysis of inorganic elements present in the fibers in the cloth is given in Table 2. Consistent with the findings of others ${ }^{12)}$, the concentration of sodium* is found to be considerably higher than that of any other metal in the treated PAN fibers.

\footnotetext{
* In discussions with JPL personnel, they report finding wide variations in sodium content in PAN fibers. It is to be emphasized that our analysis was on just one batch of T-300 fiber cloth. Variations in sodium content in PAN may be due to such variables as: type of initiator used in polymerization, whether a sodium compound was present during the PAN spinning operation, and cation content in the wash water.
}

Table 2 Inorganic elements present in T-300 fiber cloth.

\begin{tabular}{cc}
\hline Element & Amount, wt $\%$ \\
\hline $\mathrm{Na}$ & 0.2 \\
$\mathrm{~K}$ & 0.015 \\
$\mathrm{Ca}$ & 0.03 \\
$\mathrm{Ba}$ & 0.003 \\
$\mathrm{Fe}$ & 0.02 \\
$\mathrm{Mg}$ & 0.004 \\
$\mathrm{Zn}$ & 0.01 \\
$\mathrm{Ti}$ & 0.003 \\
$\mathrm{Al}$ & 0.01 \\
$\mathrm{Si}$ & 0.03 \\
\hline
\end{tabular}

Not detected: $\mathrm{B}, \mathrm{Sb}, \mathrm{Pb}, \mathrm{Ge}, \mathrm{In}, \mathrm{Bi}, \mathrm{V}, \mathrm{Ni}, \mathrm{Co}$, $\mathrm{Be}, \mathrm{Sr}, \mathrm{Ce}, \mathrm{La}, \mathrm{Zr}, \mathrm{Y} . \mathrm{Mn}, \mathrm{Cr}$, $\mathrm{Ag}, \mathrm{Cd}, \mathrm{Sn}$.

\subsection{Oxidative Etching}

Different weights of cloth were refluxed in $54 \%$ $\mathrm{HNO}_{3}$ solution (normality of 11.7 ) at about $388 \mathrm{~K}$ for $10 \mathrm{hr}$ to: (i) increase the fiber accessible total surface area and ASA and (ii) add carboxyl groups to active carbon sites. Even for the highest cloth to solution ratio used ( $30 \mathrm{mg}$ cloth/cc solution), the acid concentration of the solution was still high following oxidation; that is, it had a normality of 10.2. Oxidized samples were washed with distilled water under refluxing conditions for $40 \mathrm{hr}$ and then dried at $383 \mathrm{~K}$.

\subsection{Ion Exchange}

Replacement of hydrogen on the carboxyl groups of the $\mathrm{HNO}_{3}$-oxidized fibers by metal cations was accomplished by shaking about $0.25 \mathrm{~g}$ of fiber cloth in a $50 \mathrm{cc} 2.0$ molar solution of metal acetate in a $\mathrm{N}_{2}$ atmosphere at $343 \mathrm{~K}$ for various periods of time. The procedure is described in more detail elsewhere ${ }^{13)}$. Following exchange, the samples were washed either in flowing, cold distilled water or by refluxing in hot distilled water for varying periods of time.

\subsection{Reactivity Measurements}

Reactivity of fiber cloth samples to $0.1 \mathrm{MPa}$ of dried air was measured using a Fisher Model 460 TGA unit. About $5 \mathrm{mg}$ of sample were held in a platinum pan, suspended from a Cahn electrobalance. In all cases, samples were first heated to $383 \mathrm{~K}$ at a rate of $20 \mathrm{~K} / \mathrm{min}$ in $\mathrm{N}_{2}$ and held for $30 \mathrm{~min}$ to remove weakly adsorbed water. For 
reactivity runs at $573 \mathrm{~K}$, heating was continued in $\mathrm{N}_{2}$ at $20 \mathrm{~K} / \mathrm{min}$ up to temperature and held for $30 \mathrm{~min}$ before switching to air. Rates of burn-off at $573 \mathrm{~K}$ were expressed on a dry-ash free basis, that is following removal of water at $383 \mathrm{~K}$. Gas flow rate through the reactor and sample weight in the platinum pan were such that an external gas mass transport effect on reactivity was absent ${ }^{2}$.

\section{RESULTS AND DISCUSSION}

\subsection{Nitric Acid Oxidized Fiber Cloth}

Oxidation of carbon fiber cloth in nitric acid decreased the level of inorganic impurities, increased surface area, reduced cloth reactivity to air, but produced a negligible change in $L_{c}$ or interlayer spacing within the crystallite. For example, oxidation for $10 \mathrm{hr}$, using a cloth to $\mathrm{HNO}_{3}$ ratio of $6.0 \mathrm{mg} / \mathrm{cc}$, reduced the sodium concentration from 0.2 to $0.03 \mathrm{wt} \%$, calcium below $0.03 \%$, and barium below $0.003 \%$.

As seen in Table 3, surface area development depends upon the ratio of weight of fiber cloth oxidized to volume of acid used for a $10 \mathrm{hr}$ oxidation time. Surface areas were measured using $\mathrm{Kr}$ adsorption at $77 \mathrm{~K}$ and the BET equation with $30 \mathrm{~min}$ allowed for equilibration of each adsorption point. Outgassing temperatures between 383 and $573 \mathrm{~K}$ prior to adsorption had a negligible effect on $\mathrm{Kr}$ adsorption. The particularly large increase in surface area in going from a cloth to acid ratio of 6.6 to $6.0 \mathrm{mg} / \mathrm{cc}$ was accompanied by a significant change in the chemical analysis of the fiber cloth. That is, the original cloth and those oxidized using cloth to acid ratios of 6.6 and greater had carbon contents between 93.2 and $88.4 \%$, nitrogen contents between 6.3 and $6.8 \%$, negligible hydrogen and sulfur, and the remainder oxygen and water. The cloth oxidized using a cloth to acid ratio of 6.0 had the following analysis: $\mathrm{C}, 70.9 \% ; \mathrm{N}, 4.9 \% ; \mathrm{H}, 0.73 \%$; and balance

Table 3 Surface area development in T-300 fiber cloth upon $\mathrm{HNO}_{3}$ oxidation.

\begin{tabular}{ccc}
\hline Sample & $\begin{array}{c}\text { Cloth to Acid } \\
\mathrm{mg} / \mathrm{cc}\end{array}$ & $\begin{array}{c}\text { Surface Area } \\
\mathrm{m}^{2} / \mathrm{g}\end{array}$ \\
\hline $\mathrm{A}$ & 30 & 1.0 \\
$\mathrm{~B}$ & 25 & 1.2 \\
$\mathrm{C}$ & 6.6 & 1.5 \\
$\mathrm{D}$ & 6.0 & 2.3 \\
\hline
\end{tabular}

23.5\%. Obviously there is a large increase in oxygen and water content in this sample; but oxidation added negligible additional nitrogen to the cloth, for example as nitro groups.* Heating the sample to $383 \mathrm{~K}$ in $\mathrm{N}_{2}$ resulted in a weight loss of $11 \%$ which is attributed primarily to release of water which was hydrogen bonded to oxygen functional groups on the fiber cloth surface. Further heating of the sample to $573 \mathrm{~K}$ in $\mathrm{N}_{2}$ resulted in an additional weight loss of $10 \%$ of the dried fiber cloth, which is attributed primarily to loss of $\mathrm{CO}_{2}$ following decomposition of carboxyl groups from the fiber surface ${ }^{15}$ ).

Assuming that $12.5 \%$ of the oxidized fiber cloth (Sample D) is oxygen in the form of carboxyl groups and that each carboxyl group occupies an area of $0.083 \mathrm{~nm}^{2}$ (associated with an active site in the prismatic surface of a carbon crystallite), the area occupied by carboxyl groups is estimated to be $195 \mathrm{~m}^{2} / \mathrm{g}$. This area is considerably in excess of that measured by $\mathrm{Kr}$ adsorption at $77 \mathrm{~K}$ on this sample and suggests molecular sieving ${ }^{16)}$. That is, $\mathrm{HNO}_{3}$ at $388 \mathrm{~K}$ was able to penetrate significant pore volume which was inaccessible to $\mathrm{Kr}$ at $77 \mathrm{~K}$. Or possibly $\mathrm{HNO}_{3}$ gained access by swelling the fiber. That oxidized fiber cloth sample D was a molecular sieve was further confirmed by measuring $\mathrm{CO}_{2}$ uptake at $298 \mathrm{~K}$. From the DubininPolanyi plot ${ }^{17)}$, a micropore surface area of 139 $\mathrm{m}^{2} / \mathrm{g}$ is estimated, again a value considerably in excess of the area measured by $\mathrm{Kr}$ adsorption at $77 \mathrm{~K}$.

Reactivity of the as-received fiber cloth in air at $573 \mathrm{~K}$ was very low; that is less than $1 \%$ weight loss in $10 \mathrm{hr}$ and less than $4 \%$ weight loss in $70 \mathrm{hr}$. Reactivity of the $\mathrm{HNO}_{3}$-oxidized sample $\mathrm{D}$ was still lower, that is $0.6 \%$ in $10 \mathrm{hr}$. Presumably this decrease in reactivity can be attributed to reduction in inorganic impurity content over that of the as-received cloth.

\subsection{Ion Exchanged Fiber Cloth}

In exploratory studies, potassium, sodium, calcium, and barium were exchanged onto the

* The latter finding is consistent with results of Deno who reports that a significant number of nitro groups are added to aromatic systems only when reacted with nitric acid at concentrations considerably in excess of the $54 \mathrm{wt} \%$ used in this study ${ }^{14)}$. 
fiber cloth surface. As expected ${ }^{3,18)}$, they all catalyzed cloth reactivity to air at $573 \mathrm{~K}$. However, for each cation, reactivity did not increase monotonically with increased loading; instead it went through a maximum. This would not be expected if the metal was added only by ion exchange ${ }^{8)}$. It was thought that some metal may have been derived from the metal acetate solution which was not completely removed from the cloth pore structure by washing. A more thorough study was performed using potassium, which is described herein, in an attempt to understand relations between cloth reactivity and how the metal was added.

In the first set of experiments, the cloth which had been oxidized using a cloth to $\mathrm{HNO}_{3}$ ratio of $30 \mathrm{mg} / \mathrm{cc}$ (Sample A) was exchanged in a potassium acetate solution (starting $\mathrm{pH}$ of 8.4 ) for $50 \mathrm{hr}$. The sample was then washed by refluxing in hot distilled water for varying lengths of time to remove potassium from the pore system. Figure 1 presents results for the amount of potassium remaining on the cloth as a function of washing time. It is clear that metal loading will be a function of not only the extents of oxidation in $\mathrm{HNO}_{3}$ and subsequent cation exchange but also the severity of final washing. The curve in Figure 1 is characteristic of two concurrent processes of significantly different rates. It is suggested that the rapid process involves removal of the acetate

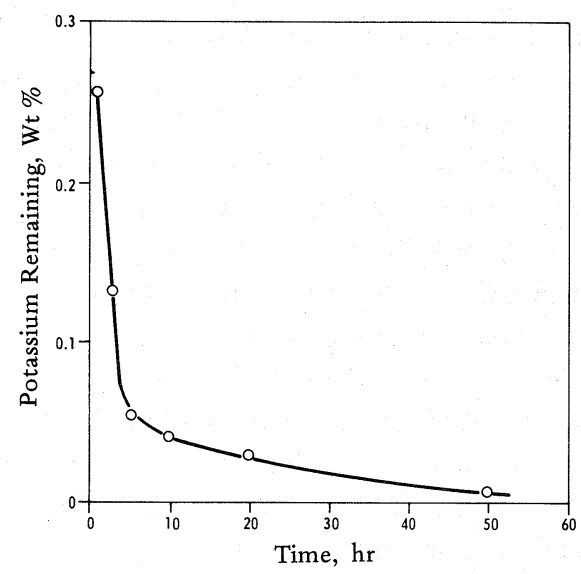

Figure 1. Effect of time of washing by refluxing in hot water on potassium content in cloth sample A.

Sample A previously exchanged with potassium acetate for $50 \mathrm{hr}$. from the pore system and the slow process involves back-ion exchange of hydrogen from hot water replacing potassium on the carboxyl groups. Extrapolation of the lower curve back to the ordinate yields a potassium concentration on the cloth at zero time of about $0.07 \%$, which is taken as the maximum value of cation loading under the conditions of previous cloth oxidation and ion exchange used. It is concluded that following washing by refluxing for about $5 \mathrm{hr}$ essentially all potassium derived from the acetate has been removed from the pore system and the potassium remaining is associated with carboxyl groups. For refluxing times progressively shorter than $5 \mathrm{hr}$, the contribution of potassium derived from the acetate to total potassium present increases sharply.

If we reason that the rate of back-ion exchange is more temperature dependent (that is, has a higher activation energy for its rate constant) than the rate of washing of the acetate from the pore system, then the use of cold water should separate the effects of these two processes still better than hot water. Thus washing of the cation exchanged cloth was also conducted in a continuous flow of fresh cold water (where an acetate ion concentration was not allowed to build up in the wash water as with refluxing). Following washing in cold water for $1 \mathrm{hr}$, the potassium concentration on the cloth was $0.078 \%$, suggesting that essentially all acetate was washed out of the pore system but that little potassium cation associated with carboxyl groups had been removed.

Reactivity plots for the fiber cloth samples containing amounts of potassium varying from 0.006 to $0.265 \%$ are shown in Figure 2 for reaction times up to $8 \mathrm{hr}$. In some cases duplicate runs were made; in these cases data points give the average values and bars give the spread in values. Even though there is a monotonic increase in reactivity with increasing potassium loading, it is obvious that the specific activity of the catalyst decreases at higher loadings, as seen in Figure 3 where burnoff following reaction for $8 \mathrm{hr}$ is plotted against potassium loading. This could be due to at least three reasons: (i) decreasing dispersion of the exchanged potassium with increasing concentration, (ii) poorer dispersion of the potassium derived from potassium acetate than that derived from the exchanged cation, and (iii) blockage of 


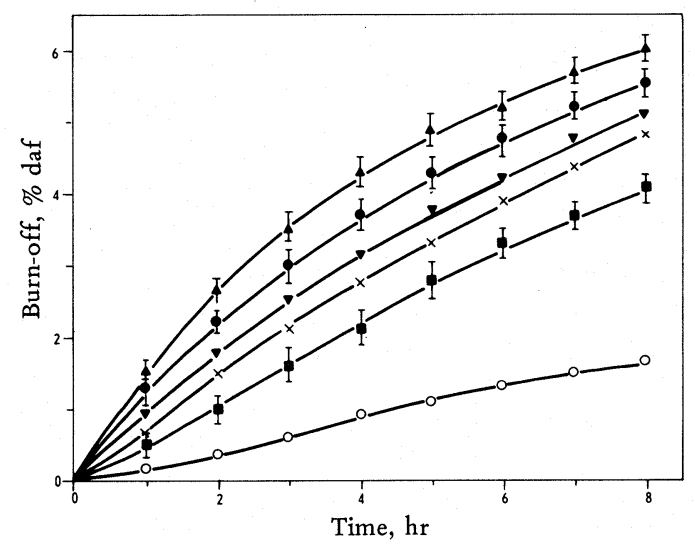

Figure 2. Reactivity plots of cloth sample A in air at $573 \mathrm{~K}$ for various potassium loadings, in wt \%: $\bigcirc, 0.006 ; \square, 0.031$; $\times, 0.045 ; \quad \nabla, 0.051 ; \quad 0,0.133$; $\mathbf{\Delta}, 0.265$.

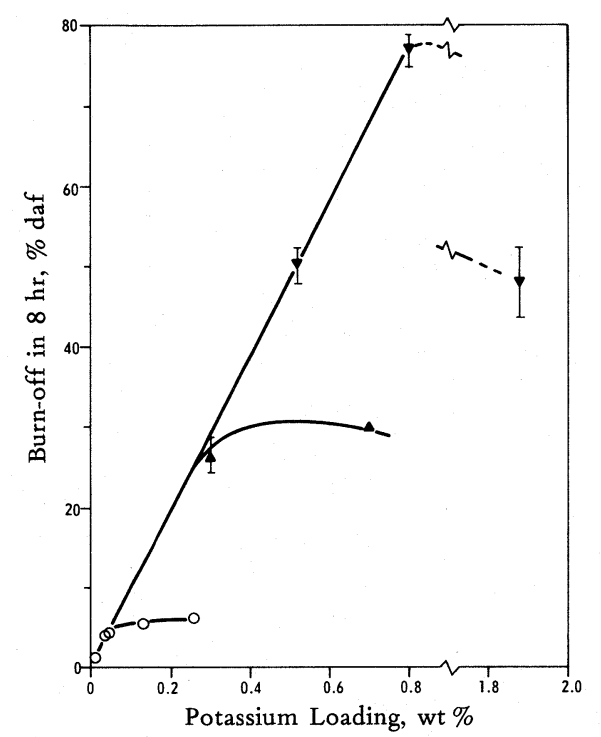

Figure 3. Extent of burn-off in $8 \mathrm{hr}$ in air at $573 \mathrm{~K}$ of cloth samples loaded with varying amounts of potassium.

Sample: $O$, A; $\boldsymbol{\Delta}, \mathrm{C} ; \boldsymbol{\nabla}, \mathrm{D}$.

some of the pore system. Krypton surface areas of samples loaded to 0.133 and $0.265 \%$ potassium actually increased $\left(1.9 \mathrm{~m}^{2} / \mathrm{g}\right)$ over that of the oxidized fiber $\left(1.0 \mathrm{~m}^{2} / \mathrm{g}\right)$, and thus pore blockage at this level of loading was not present. The possibility that the degree of dispersion of the metal (or its oxide) is different following decomposition of the metal carboxylate which is bonded to the surface compared to decomposition of metal acetate particles (agglomerates) sitting on the surface is interesting to contemplate. A definitive answer does not come from this study, but is an important question when considering catalyst addition to carbon substrates and subsequent catalyst efficiency.

In additional experiments, cloths oxidized using cloth to $\mathrm{HNO}_{3}$ ratios of 6.6 and $6.0 \mathrm{mg} / \mathrm{cc}$ were exchanged with potassium acetate. For sample $\mathrm{C}$, exchange was conducted for $30 \mathrm{hr}$, followed by washing for $1 \mathrm{hr}$ using either refluxing in hot water or flowing cold water. Concentrations of potassium remaining in the fiber were 0.71 and $0.31 \%$, respectively. It is suggested, as just discussed, that the loading of $0.30 \%$ essentially represents potassium derived from carboxyl groups and that the loading of $0.71 \%$ represents potassium derived both from carboxyl and acetate entities. Enhanced loading from these two sources onto cloth sample $C$ over that found on exchanged cloth sample $A$ is consistent with the larger surface area (and pore volume) in the former sample.

The reactivity plot for the $0.30 \%$ potassium loaded sample is shown in Figure 4 . Over the $8 \mathrm{hr}$ reaction period, reactivity continuously decreases with increasing reaction time despite a build-up in catalyst concentration in the carbon remaining. This suggests that: (i) increasing agglomeration (sintering) of catalyst particles is occurring with increasing burn-off, that is catalyst dispersion is decreasing and/or (ii) the catalyst is losing contact with the carbon surface. McKee and Chatterji have shown that the mechanism of catalysis of carbon gasification by potassium involves oxidation-reduction steps ${ }^{19)}$. That is, the higher oxide $\mathrm{K}_{2} \mathrm{O}_{2}$ is reduced by the carbon to $\mathrm{K}_{2} \mathrm{O}$ which, in turn, is reoxidized to $\mathrm{K}_{2} \mathrm{O}_{2}$ by air. The rate of agglomeration is dependent upon the mobility of the catalyst species on the surface. Even though reaction temperature was below the melting point of $\mathrm{K}_{2} \mathrm{O}_{2}(663 \mathrm{~K})$, Thomas and Walker ${ }^{20}$ ) have shown that solid catalyst particles can have high mobility on carbon surfaces, particularly in an oxygen environment. Further, Baker has shown, from in-situ electron microscopy studies, that species on carbon surfaces in an $\mathrm{O}_{2}$ atmosphere commence having significant mobility above their Tammann temperature, that is one half their melting temperature, expressed in degrees $\mathrm{K}^{21)}$. 


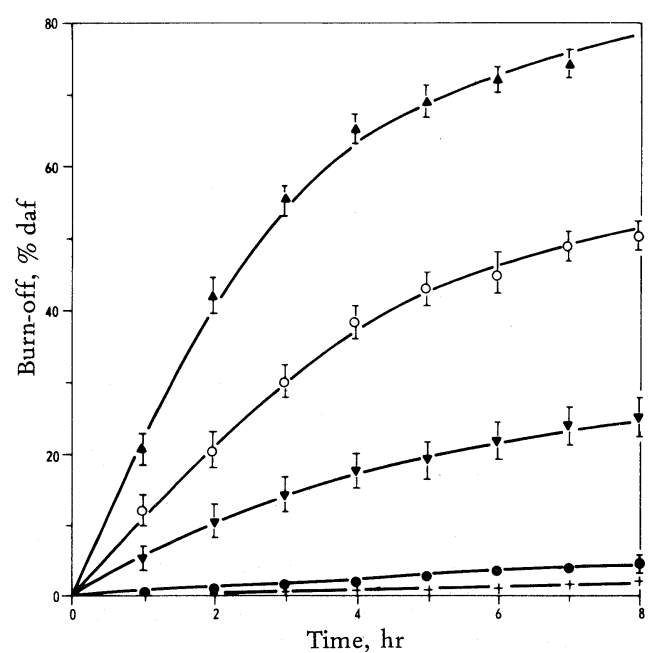

Figure 4. Reactivity plots of cloth samples with various potassium loadings in air at $573 \mathrm{~K}$.

Sample: +, A-0.006\% K; O, A-0.031\% K;

$\boldsymbol{\nabla}, \mathrm{C}-0.30 \% \mathrm{~K} ; \mathrm{O}, \mathrm{D}-0.52 \% \mathrm{~K}$; $\boldsymbol{\Delta}, \mathrm{D}-0.79 \% \mathrm{~K}$.

Thus, at a reaction temperature of $573 \mathrm{~K}, \mathrm{~K}_{2} \mathrm{O}_{2}$ (having a Tammann temperature of $332 \mathrm{~K}$ ) would be expected to have significant mobility on carbon surfaces.

Burn-offs in $8 \mathrm{hr}$ for the samples loaded to 0.30 and $0.71 \%$ potassium are given in Figure 3. As with cloth sample A, it is suggested that there is an optimum potassium loading to achieve maximum cloth reactivity for Sample C. Additional loading increases reactivity only slightly or may, in some cases, even decrease reactivity, as will now be seen.

For sample D, exchange was conducted for 10 , 30 , and $50 \mathrm{hr}$ in potassium acetate followed by washing in running cold water for $1 \mathrm{hr}$. Loadings of potassium were $0.52,0.79$, and $1.8 \%$, respectively. These results suggest that transport of the acetate solution into and out of the fiber cloth pore system was slow. This is consistent with this oxidized sample having some molecular sieve character, as previously discussed. Figure 4 presents reactivity plots for the samples loaded to 0.52 and $0.79 \%$ potassium. Again reactivities decrease continually with increasing time of reaction. Burn-offs in $8 \mathrm{hr}$ for all samples are given in Figure 3. For sample D, again an optimum potassium loading is suggested; at higher loadings there is a significant decrease in cloth reactivity, probably due to pore blockage and/or coverage of the carbon surface with more than a monolayer of potassium. That is, washing the sample, which was exchanged for 50 $\mathrm{hr}$, in running cold water for $1 \mathrm{hr}$ appears to have not removed all potassium acetate from the pore system. If optimum loading is taken as about $0.79 \%$ potassium and if it is assumed that each potassium atom was previously associated with a carboxyl group, it is calculated that the potassium carboxylate occupied about $10 \mathrm{~m}^{2} / \mathrm{g}$ of surface. Again this is larger than the $\mathrm{Kr}$ area of oxidized cloth sample D and is consistent with molecular sieving. However, the area is much less than that which was estimated to be occupied by carboxyl groups following nitric acid oxidation (that is, $195 \mathrm{~m}^{2} / \mathrm{g}$ ). This suggests that: (i) potassium only exchanged on a small fraction of the carboxyl groups following oxidation, (ii) oxidation in nitric acid added both carboxyl, acidic phenolic and lactone groups to the fiber surface, and/or (iii) nitric acid was not completely washed from the pore system.

\section{CONCLUSIONS}

The reactivity of $\mathrm{PAN}$ fiber cloth to air can be substantially increased by the addition of metals to the cloth via ion exchange from metal acetate solutions. However, since the surface area of PAN fibers (even those heated to less than $1800 \mathrm{~K}$ ) is very low, significant metal addition via ion exchange can only be achieved if the fiber is first treated with nitric acid to increase surface area and, as importantly, to add carboxyl groups. The amount of metal which can be added to the fiber is a function of the severity of oxidative treatment, the time of subsequent exchange, and the degree of subsequent removal of metal from the fiber by washing. Fiber cloth reactivity to air appears to increase essentially linearly with increase in potassium loading via ion exchange. Additional potassium loading originating from potassium acetate left in the pore system of the fibers following washing appears to increase reactivity only slightly in some cases and in other cases to, in fact, result in reduction in fiber cloth reactivity. It is suspected that there will be an optimum in the extent of fiber oxidation to achieve enhanced reactivity. At the optimum, both fiber cloth 
reactivity and mechanical properties of composites made therefrom will be enhanced. Too much fiber oxidation will lead to an unacceptable reduction in mechanical properties of the composites subsequently fabricated, even though fiber reactivity to air may be further enhanced.

Acknowledgements - This research was supported by the Jet Propulsion Laboratory California Institute of Technology on Contract NO. 955236. Helpful Discussions with W. Dowler and K. Ramohalli are sincerely appreciated.

\section{REFERENCES}

1) S. E. Wentworth, A. O. King, and R. J. Shuford, Army Materials \& Mechanics Research Center Report No. TR79-1, January, 1979.

2) P. L. Walker, Jr., F. Rusinko, Jr., and L. G. Austin, Advances in Catalysis (Edited by D.D. Eley, P.W. Selwood, and P. B. Weisz), Vol. 11, pp. 133-221, Academic Press, New York (1959).

3) P. L. Walker, Jr., M. Shelef, and R. A. Anderson, Chemistry and Physics of Carbon (Edited by P. L. Walker, Jr.), Vol. 4, pp. 287380, Marcel Dekker, New York (1968).

4) D. W. McKee and V. J. Mimeault, Chemistry and Physics of Carbon (Edited by P.L. Walker, Jr. and P. A. Thrower), Vol. 8, pp. 151-245, Marcel Dekker, New York (1978).

5) C. S. Brooks, G. S. Golden, and D. A. Scola, Carbon 12, 609 (1974).
6) B. Rand and R. Robinson, Carbon 15, 257 (1977).

7) B. Rand and R. Robinson, Carbon 15, 311 (1977).

8) E. J. Hippo, R. G. Jenkins, and P. L. Walker, Jr., Fuel 58, 338 (1979).

9) Technical Information Bulletin No. 465-223, Union Carbide Corp.

10) S. C. Bennett and D. J. Johnson, Carbon 17, 25 (1979).

11) W. Watt, Carbon 10, 121 (1972).

12) L. T. Drzal, Carbon 15, 129 (1977).

13) H. N.S. Schafer, Fuel 49, 197 (1970).

14) N. C. Deno, The Pennsylvania State University, Private Communication, November, 1979.

15) H. N. S. Schafer, Fuel 58, 673 (1979).

16) P. L. Walker, Jr., L.G. Austin, and S. P. Nandi, Chemistry and Physics of Carbon (Edited by P.L. Walker, Jr.), Vol. 2, pp. 257-371, Marcel Dekker, New York (1966).

17) M. M. Dubinin, Chemistry and Physics of Carbon (Edited by P. L. Walker, Jr.), Vol. 2, pp. 51-120, Marcel Dekker, New York (1966).

18) P. L. Walker, Jr., O. P. Mahajan, and M. Komatsu, Preprints American Chemical Society, Division Fuel Chemistry Meeting, Vol. 24, No. 3, 10 (1979).

19) D. W. McKee and D. Chatterji, Carbon 13, 381 (1975).

20) J. M. Thomas and P. L. Walker, Jr., J. Chem. Phys. 41, 587 (1964).

21) R. T. K. Baker, Exxon Corp., Private Communication, January, 1980. 